\title{
Research and Implementation of Measurement Data Wavelet De-noising and 3D Visualization of Farmland
}

\author{
Weidong Zhuang, Chun Wang, and Xi Wang \\ College of Engineering, Heilongjiang Bayi Agriculture University, \\ 163319, Daqing, Heilongjiang Province, China \\ $81 \mathrm{nda} 163 . \mathrm{com}$
}

\begin{abstract}
The precision agriculture decision-making needs of geographic information visualization analysis for farmland. In order to get 3D visualization of farmland, the Matlab software and wavelet transform were used for 3Dterrain survey data processing. Haar, DB6, Sym8, coif3 wavelet were used on the 3 layer decomposition of elevation data de-noising test, DB6 wavelet was used on the two to seven layer decomposition de-noising. The measurement plane scatter plots, contour map, 3D surface chart of the farmland was drew. The research shows that using a db6 wavelet five level decomposition denoising for elevation data measured by DGPS continuously was suitable for the 3D-terrain farmland surveying and mapping in which farmland have the more moderate wavy. This may well reflect the farmland terrain features.
\end{abstract}

Keywords: Precision agriculture, farmland, Wavelet de-noising, 3D visualization.

\section{Introduction}

The goal of precision agriculture (PA) is the management of crop and soil variability to increase profitability and reduce environmental impact. The PA decision-making needs of geographic information visualization analysis for farmland. The measurement of elevation data for sloping farmland's 3D topography uses real-time differential GPS system in order to reduce the cost of measuring equipment and make it convenient and easy. The positioned GPS observation is a set of sequence related to time and the discretization of time functions. GPS measurement error contains the ionosphere delay, troposphere delay, multi-path effect, receiver software, hardware and so on. The difference technique can eliminate most of the system error except for random measurement error that belongs to the high-frequency measurement noise. If the high frequency measurement noise was removed, it can reduce the disturbance of positioning precision, which is caused by measurement noise, as a result of improving the accuracy and reliability of the GPS.

The real-time differential GPS system was used to gather 3D topography information for farmland in the field of No. 4-3-1-4, Sinograin northern agriculture 
development Co, LTD. Nenjiang County, Heilongjiang Province, China on April 27th, 2011. The farmland is the typical sloping field and its square is $61.4 \mathrm{hm}^{2}$. The measuring speed is between 20 to 30 kilometers per hour. It is equipped with AgGPS332 receiver that can receive the signal of Satellite Based Augmentation Systems (SBAS). The GPS position data was recorded by computer once per second, 1302 points were collected. The thesis researches the measurement data processing and visualization of farmland's 3D topography.

\section{Measurement Data Wavelet De-Noising}

Wavelet is the wave of small area, limited length and zero mean value. Wavelet transformation is the space frequency of localization analysis. It takes signal to Multiscale Analysis through the idea of dilation and translation in order to achieve the applied time in both high and low frequency and get the analysis in both time and frequency domain. It also can be focused to the signal in any detail, thus extracting information effectively. It is called "Math microscope". At present the analysis of wavelet is widely used in Mathematics study, signal analysis, image processing, classification and recognition, synthetic music and language, medical imaging and diagnosis and so on.

\subsection{Principle of Wavelet Noise Elimination}

It usually contains non-use signal gathering Data signals from the computer, which is called noise signal. The signal noise elimination prevents non-use part of signal Elimination and reserves useful part of signal enhancement. A d signal model that contains noise shows in this form:

$$
\mathrm{s}(\mathrm{k})=\mathrm{f}(\mathrm{k})+\sigma \cdot \mathrm{e}(\mathrm{k}) \quad \mathrm{k}=0,1, \ldots, \mathrm{n}-1
$$

In the formula, $\mathrm{f}(\mathrm{k})$ is useful signal, $\mathrm{e}(\mathrm{k})$ is noise, $\mathrm{s}(\mathrm{k})$ is signal including noise. In fact, $\mathrm{f}(\mathrm{k})$ is usually low frequency or some stable signal, $\mathrm{e}(\mathrm{k})$ is usually high frequency. So there are three procedures to deal with noise elimination for a d signal. (1): It resolve Original signal into wavelet so that the noise usually belongs to high frequency coefficient. (2): It takes quantization process for high frequency coefficient of Wavelet decomposition in some form, such as threshold. (3): It takes reconstruction of a d wavelet for the bottom of low frequency coefficient and high frequency coefficients of every layer of Wavelet decomposition in order to eliminate noise.

\subsection{Choice of Wavelet Basis Function}

The process of reducing the noise of signal is a operation that the signal is decomposed and restructured. We should search for a set of functional form that stands for the features of the signal.

Wavelet function is an infinite number as well as wavelet basis function. That is because different wavelet basis function has different characteristics of the time 
frequency. And different wavelet basis function may come out different results when using the same method to analysis problem. We choose the proper wavelet basis according to the characteristics and application of wavelet function in practice.

Choosing wavelet basis we should consider its Mathematics characteristics including support, symmetry, orthogonality, regularity, vanishing moments and wavelet itself. There are Common wavelet basis in the Matlab toolkit including haar, dbN, symN, CoifN.

The premises of GPS data processing of changing wavelet are discrete values observed quantity. So wavelet basis function has the characteristics of Discrete wavelet transformation and orthogonality, symmetry. In order to position GPS data processing fast and precisely we could choose wavelet basis function, such as Haar, $\mathrm{dbN}$, symN, CoifN.

\subsection{Wavelet De-noising}

In order to study the effect of GPS data noise elimination of changing wavelet, wavelet de-noising is analyzed.

Using elevation data to the variable z1 with assignment, then Haar, DB6, Sym8, coif3 wavelet were used on the three layer decomposition. In addition, choosing threshold model of sure and scale noise to eliminate noise. The original elevation curve data and wavelet de-noising curve of elevation show in Fig.1.
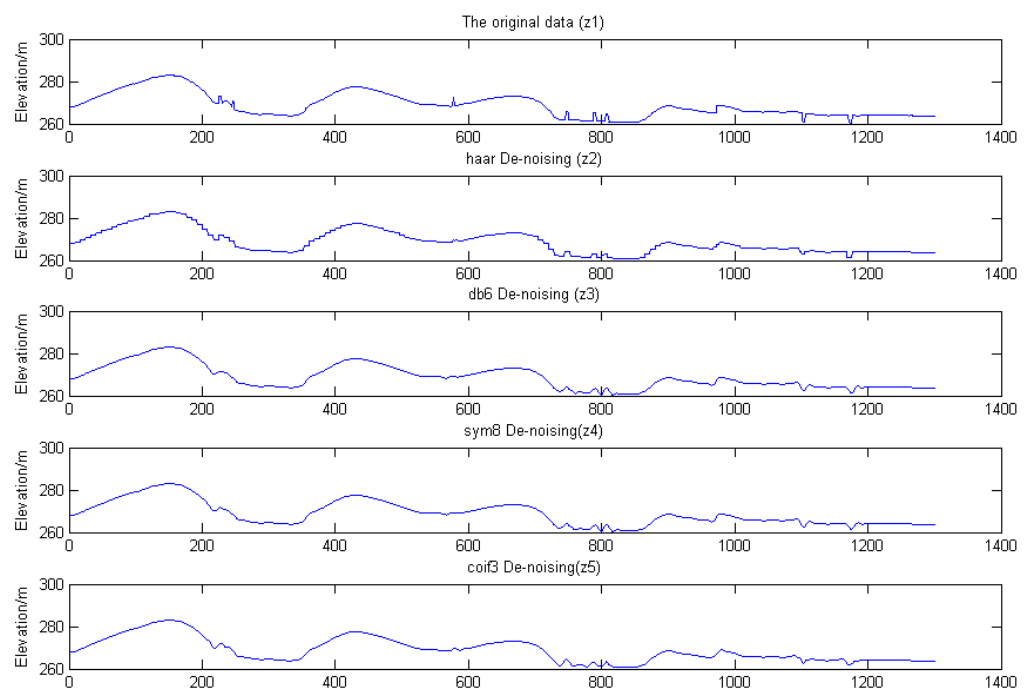

Fig. 1. Wavelet de-noising curve of elevation 
It can be seen from the Fig. 1 that the wavelet including haar, db6, sym8, coif3 has an effect on noise elimination and filter out outliers. Wavelet Db6 and sym8 for noise elimination is more smooth, so is the effect.

In order to study the GPS elevation curve data de-noising effect of the layering wavelet, using elevation data to the variable $\mathrm{z} 1$ with assignment, then the wavelet db6 is used from the two layers to seven layers for de-noising. The original elevation data and the wavelet db6 de-noising curve of elevation show in Fig.2.

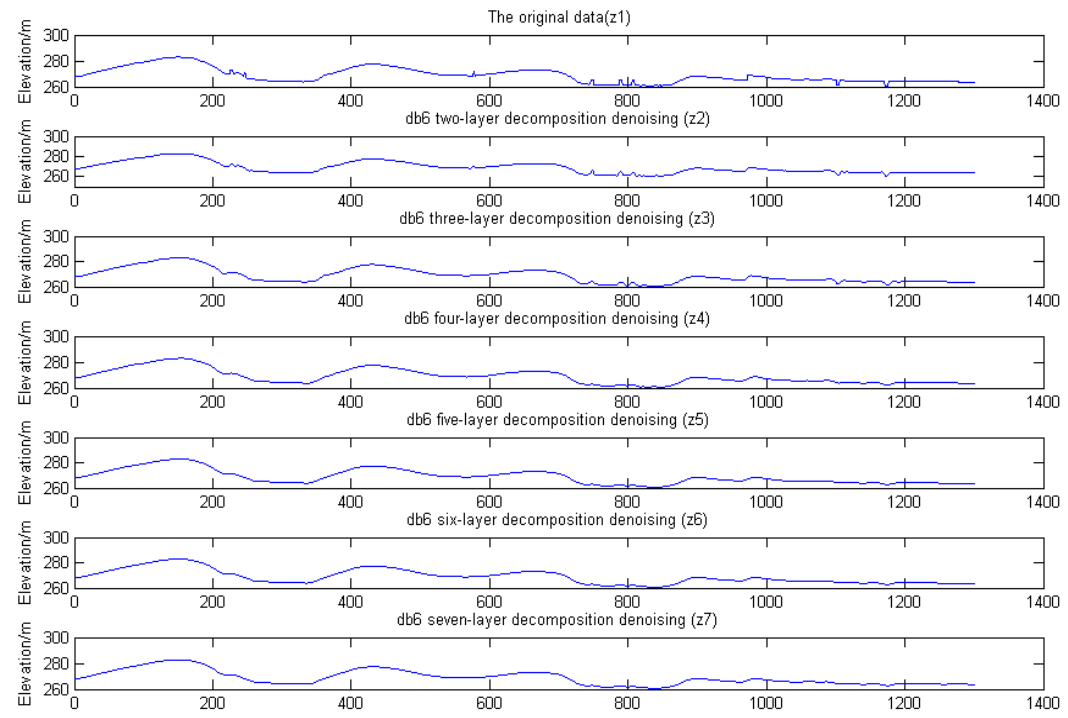

Fig. 2. The db6 wavelet de-noising using multilayer level for elevation

It can be seen from the Fig. 2 that the more layers and more smooth after wavelet de-noising. However, with the increasing of layers, the more detail data lost. For example, the seven layers wavelet de-noising is too much smooth. The five layers wavelet de-noising reserves the trend of changing data, and the curve is relatively smooth.

Because slop farmland generally have more moderate undulating and field 3D topographic survey is continuous sampling, the five layers db6 wavelet de-noising can be used for farmland elevation data.

\section{Drawing Farmland's 3D Topography}

The latitude and longitude coordinates of the GPS positioning acquisition of data points in the program using the UTM projection, the transformation to the plane rectangular coordinates.

In this study, using Matlab as data processing and drawing tools. First, projection coordinates and elevation values of the collected data points were assigned to three 
variables to $\mathrm{x}, \mathrm{y}$, and $\mathrm{z}$. In the operation, export Access data to Excel, then to Matlab. In another way, assign the variable $\mathrm{x}, \mathrm{y}, \mathrm{z}$ with Matlab, then copy and paste the corresponding data series to the variable value table of Matlab. Compared with the former, the latter is that a large amount of column values fast assign the variable value of Matlab.

\subsection{Plane Scatter Plots}

In order to show each sampling point location, need to draw a plane scatter plot of the sampling points. Draw blue scatter diagram, Matlab command sentences are as follows.

plot $\left(\mathrm{x}, \mathrm{y},{ }^{\prime *} \mathrm{~b} \mathrm{O}^{\prime}\right)$;

xlabel (' $x$ coordinate');

ylabel ('y coordinate') ;

Plane scatter diagram that is made up of 1302 data points shows in Fig.3.

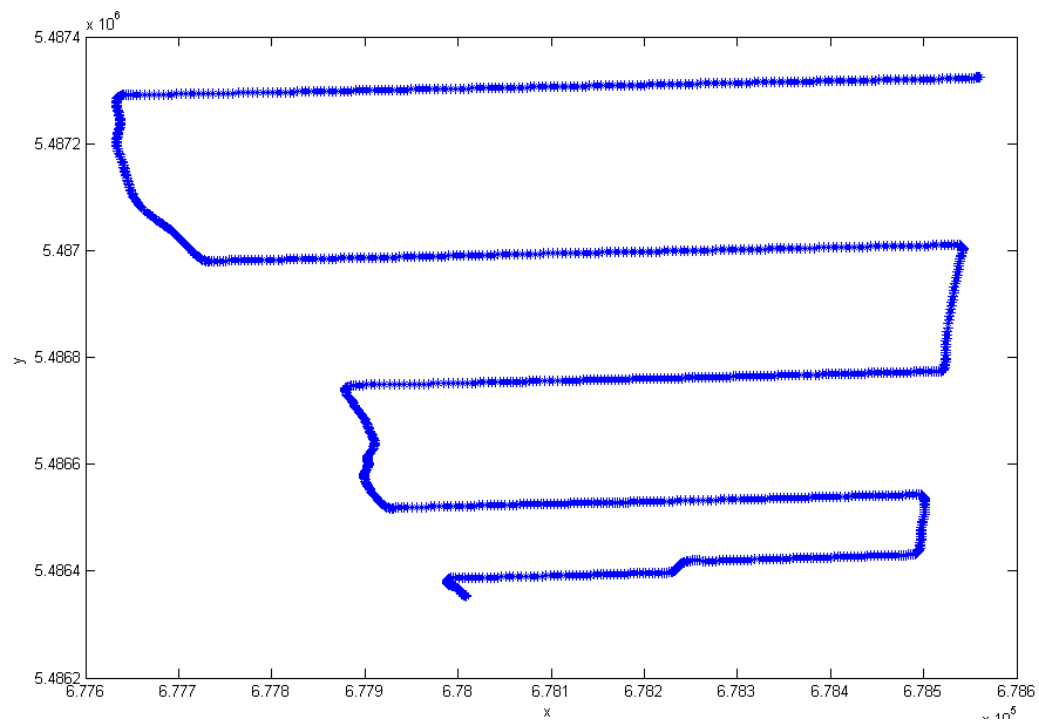

Fig. 3. The sampling points scatter diagram

\subsection{Data Interpolation}

Sampling points is an irregular distribution practically in farmland. In order to generate isoline and isosurface with Matlab, we should conduct data interpolation and gridding. Matlab command sentences of data interpolation are as follows. 
$\mathrm{X}=$ linspace $(\min (\mathrm{x}), \max (\mathrm{x}), 100)$;

$\mathrm{Y}=1$ inspace $(\min (\mathrm{y}), \max (\mathrm{y}), 100)$;

Linspace is one of the instructions that are used to produce Line vector between two points. Take 100 points from minimum to maximum in the experiments. Matlab command sentences of data gridding are as follows.

$[\mathrm{X}, \mathrm{Y}]=$ meshgrid $(\mathrm{X}, \mathrm{Y})$;

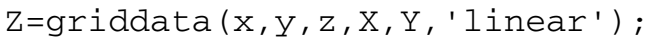

The effect of meshigrid $(\mathrm{X}, \mathrm{Y})$ is that conducting a matrix for line vector $\mathrm{X}$ and column vector $\mathrm{Y}$, generating a cartesian product space and drawing the frame through two points on the axis.

We use designated algorithm. There are four kinds. (1) 'linear': Base on the linear (2) 'cubic': triangle-based cubic interpolation; (3) 'nearest': the nearest interpolation; (4) ' $v 4$ ': Lattice point spline function interpolation algorithm. Through gathering 3D topography information for farmland, the curve that is made up of ' $\mathrm{v} 4$ ' interpolation is more smooth. But the interpolation of the four corners will be larger deviation, the curve that is made up of 'linear' interpolation is more ideal.

\subsection{Drawing Contour Map}

Drawing contour map is an important branch of visualization technology, we can achieve contour map fast with Matlab. The contour map shows in Fig.4.

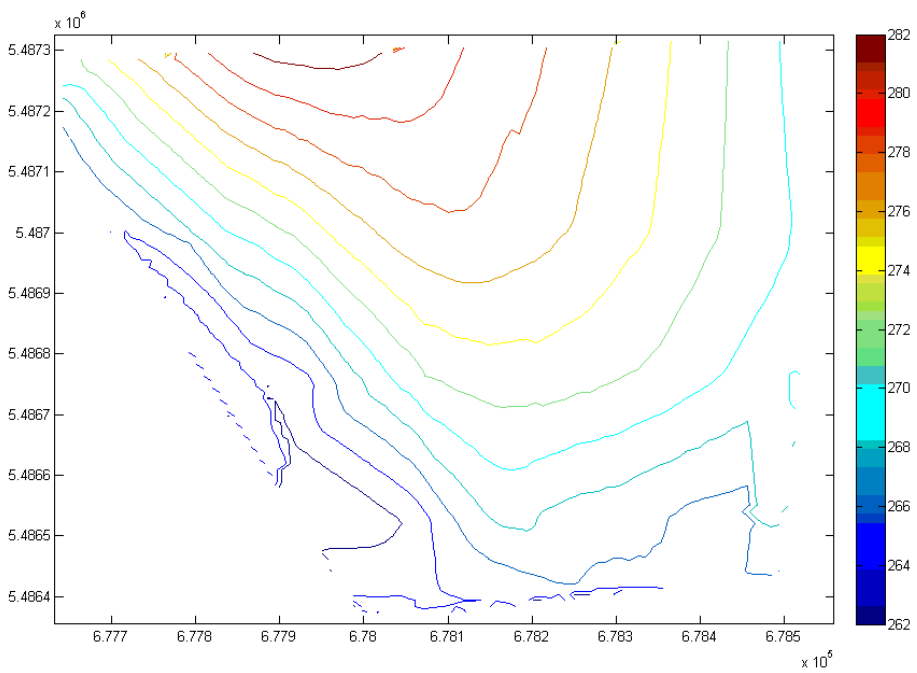

Fig. 4. The contour map of elevation 
DB6 wavelet was used on the fifth layer decomposition de-noising. Then make an interpolation. The contour map of elevation denoised shows in Fig.5. Matlab command sentences are as follows.

contour $(\mathrm{X}, \mathrm{Y}, \mathrm{Z})$;

colorbar;

z5 =wden ( $\mathrm{z}$, 'heursure' ' 's' ', one' , 5, 'db6' ) ;

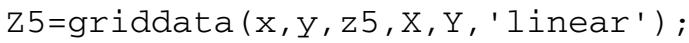

contour (X, Y, Z5) ;

colorbar;

Colorbar is used for generating illustrations.

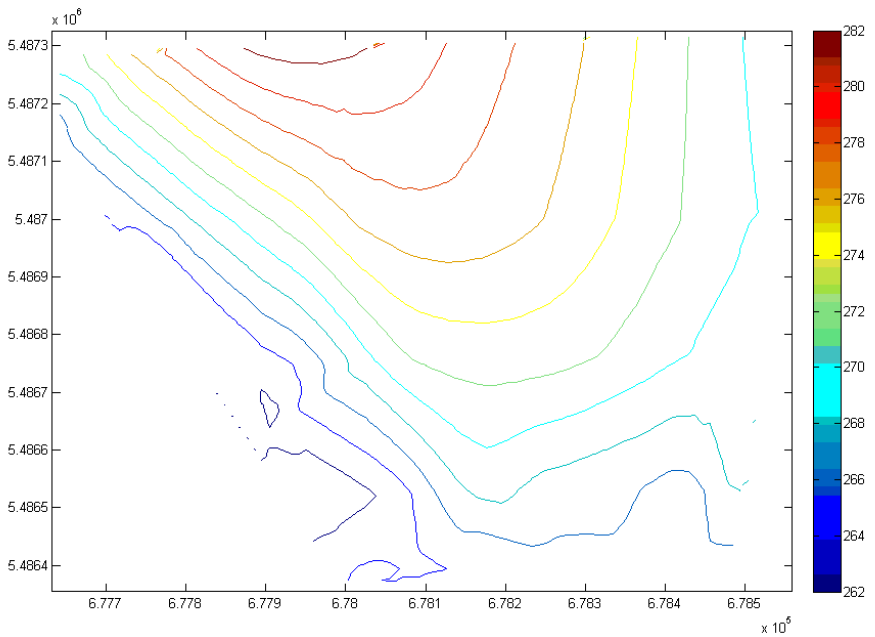

Fig. 5. The contour map of elevation denoising

It can be seen from the Fig. 4 and Fig. 5 that the contour map of elevation data denoising is better than before.

\subsection{Drawing 3-D Surface Map}

3-D surface chart can be fast achieved with Matlab. Matlab command sentences of the3-D surface chart, which is made up of interpolation, are as follows.

$\operatorname{surf}(X, Y, Z) ;$

$\operatorname{surf}(X, Y, Z 5)$;

Three-dimensional topographic surface map and three-dimensional topographic surface map of elevation de-noising show in Fig.6 and Fig.7. 


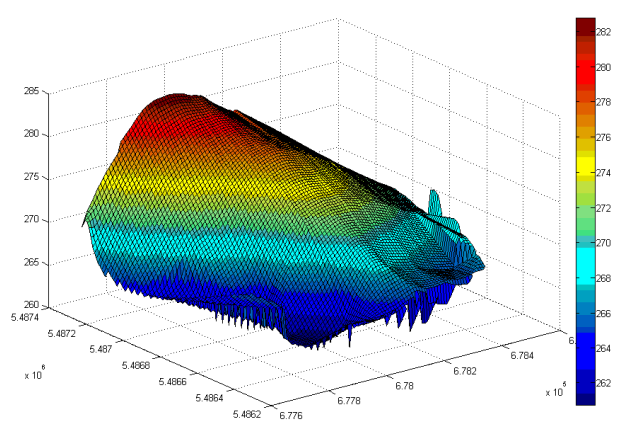

Fig. 6. Three-dimensional topographic surface map

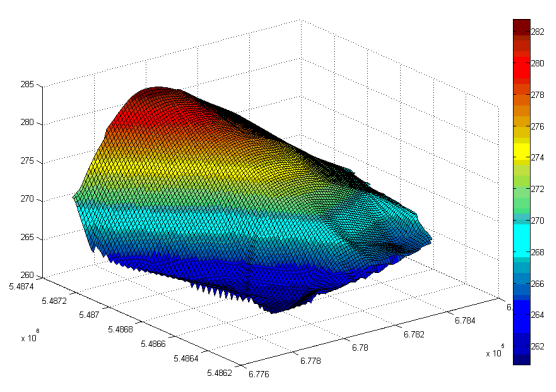

Fig. 7. Three-dimensional topographic surface map of elevation de-noising

It can be seen from the Fig. 6 and Fig.7 that three-dimensional topographic surface map of elevation de-noising is better than before. This method was suitable for the sloping farmland, which has the more moderate wavy. The wavelet db6 is used on the fifth layer for de-noising by farmland's 3D topography, and then draw 3-D surface chart. It has a good effect on drawing Geomorphology.

\section{Conclusions}

It takes the measures of wavelet transformation to process the data of farmland's 3D topography with Matlab. Haar, DB6, Sym8, coif3 wavelet were used on the 3 layer decomposition of elevation data de-noising test, DB6 wavelet was used on the $2 \sim 7$ layer decomposition de-noising. The research shows that the wavelet db6 was used on the 5 layer decomposition for de-noising, which could achieve elevation data of farmland.

The precision agriculture decision-making is widely needed in geographic information visualization analysis for farmland. Matlab has the function of Data calculation and drawing. By the technology, plane scatter plots, contour map, 3-D surface map of the farmland are drawn. 
The research shows that using the wavelet db6 5 level decomposition de-noising for elevation data measured by DGPS continuously was suitable for the 3D-terrain farmland surveying and mapping in which farmland have the more moderate wavy. This may well reflect the farmland 3D topography features.

Acknowledgments. We wish to thank twelve five years science and technology plan for rural area project No.2011BAD20B03, No.2011BAD20B06 and No.2012BAD04B01, China, for funding this research. We also wish to thank the anonymous reviewers for their useful suggestions that greatly improved this paper.

\section{References}

1. Kumhálová, J., Kumhála, F., Kroulík, M., Matějková, Š.: The impact of topography on soil properties and yield and the effects of weather conditions. Precision Agriculture 12(6), $813-830$ (2011)

2. Fountas, S., Blackmore, S., Ess, D., Hawkins, S., Blumhoff, G., Lowenberg-Deboer, J., Sorensen, C.G.: Farmer Experience with Precision Agriculture in Denmark and the US Eastern Corn Belt. Precision Agriculture 6, 121-141 (2005)

3. Asaduzzaman, K., Reaz, M.B.I., Mohd-Yasin, F., Sim, K.S., Hussain, M.S.: A Study on Discrete Wavelet-Based Noise Removal from EEG Signals. Advances in Experimental Medicine and Biology 680(pt. 6), 593-599 (2010)

4. Larson, J.A., Roberts, R.K., English, B.C., Larkin, S.L., Marra, M.C., Martin, S.W., et al.: Factors affecting farmer adoption of remotely sensed imagery for precision management in cotton production. Precision Agric. 9, 195-208 (2008)

5. Chung, G., Vese, L.A.: Energy Minimization Based Segmentation and Denoising Using a Multilayer Level Set Approach. In: Rangarajan, A., Vemuri, B.C., Yuille, A.L. (eds.) EMMCVPR 2005. LNCS, vol. 3757, pp. 439-455. Springer, Heidelberg (2005)

6. Zhuang, W.: Study on Soybean Variable Rate Fertilizing and Seeding in the Black Soil Gentle Hilly Regions of the Northeast China. Heilongjiang Bayi Agriculture University, Daqing (2011)

7. Zhuang, W.: Application of GPS \&GIS for precision agriculture. Guangming Daily Publishing Company, Beijing (2009)

8. Meng, Z., Fu, W., Liu, H.: Design and implementation of 3D topgraphic surveying system in vehicle for field precision leveling. Transactions of the CSAE 25(S2), 255-259 (2009)

9. Zhang, M., Chen, Y., Jia, W.T.: Design of three dimensional topographic information measuring system. Journal of Jilin University (Engineering and Technology Edition) 37(6), 1451-1454 (2007)

10. Zhang, Y.-L., Bai, W.-B., Jiao, X.-Y., Wang, L.-G., Wang, J.-S.: Study on 3D Visualization of Farmland Soil Nutrients Spatial Variability in Loess Area of Mining Subsidence. Journal of Shanxi Agricultural Sciences 39(8), 894-896 (2011)

11. Fu, Y., Zhang, C.: A New Seismic Data De-Noising Method Based on Wavelet Transform. In: Proceedings of the 2008 International Conference on Computer Science and Information Technology, CCSIT 2008, pp. 92-96 (2008) 